UCRL-JC-124937

PREPRINT

\title{
Producing National Ignition Facility (NIF) - Quality Beams on the Nova and Beamlet Lasers
}

C. C. Widmayer, J. M. Auerbach, R. B. Ehrlich, M. A. Henesian, J. T. Hunt, J. K. Lawson, D. Milam, P. A. Renard, D. R. Speck, P. J. Wegner, T. L. Weiland, W. H. Williams, C. R. Wolfe, B. M. Van Wonterghem

This paper was prepared for submittal to the American Nuclear Society 12th Topical Meeting on the Technology of Fusion Energy Reno, NV

June 16-20, 1996

August 20, 1996

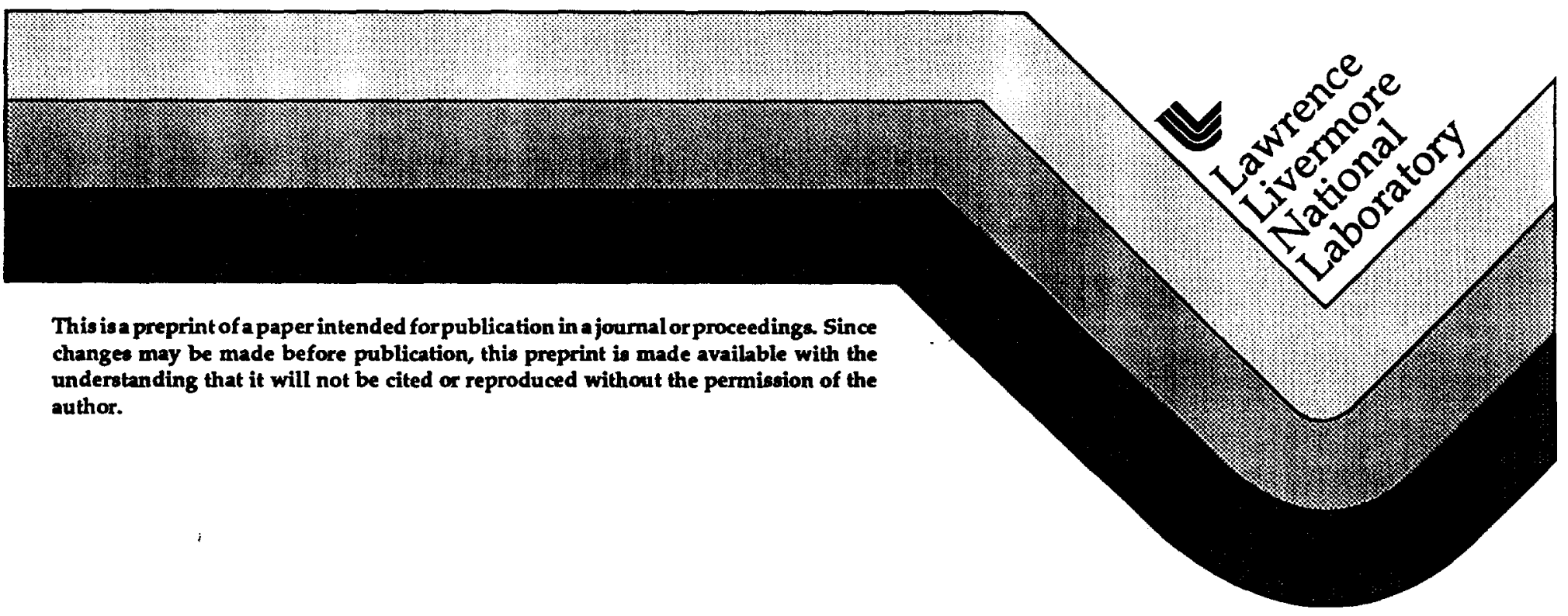




\section{DISCLAIMER}

This document was prepared as an account of work sponsored by an agency of the United States Government. Neither the United States Govemment nor the University of California nor any of their employees, make any warranty, express or implied, or assume any liability or responsibility for the accuracy, completeness, or usefulness of any information, apparatus, product, or process disclosed, or represents that its specific commercial products, process, or service by trade name, trademark manufacturer, or otherwise, does not necessarily constitute or imply its endorsement recommendation, or favoring by the United States Government or the University of California. The views and opinions of authors expressed herein do not necessarily state or reflect those of the United States Government or the University of California, and shall not be used for advertising or product endorsement purposes. 


\title{
PRODUCING NATIONAL IGNITION FACILITY (NIF)-QUALITY BEAMS ON THE NOVA AND BEAMLET LASERS
}

\author{
C. Clay Widmayer \\ Jerome M. Auerbach \\ Robert B. Ehrlich \\ Mark A. Henesian \\ John T. Hunt \\ Janice K. Lawson \\ David Milam \\ Paul A. Renard \\ D. Ralph Speck \\ Paul J. Wegner \\ Timothy L. Weiland \\ Wade H. Williams \\ C. Robert Wolfe \\ Bruno M. Van Wonterghem \\ Lawrence Livermore National Laboratory, University of California \\ Laser Programs \\ 7000 East Avenue, $L-490$ \\ Livermore, California 94551-9900 USA \\ $510-4221100$
}

\begin{abstract}
The Nova and Beamlet Lasers were used to simulate the beam propagation conditions that will be encountered during the National Ignition Facility operation. Perturbation theory predicts that there is a $5 \mathrm{~mm}$ scale length propagation mode that experiences large nonlinear power growth. This mode was observed in the tests. Further tests have confirmed that this mode can be suppressed with improved spatial filtering.
\end{abstract}

\section{INTRODUCTION}

Historically, nonlinear small scale self-focusing ${ }^{1}$ has limited the performance of high intensity solid state lasers. This phenomenon is seen in photographic images of pulses that display periodic spatial modulations, or ripples. Ripples accumulate due to imperfections on optics, diffraction effects or misalignment of the beam. At high intensities nonlinear effects conspire to amplify these ripples and if allowed to continue unchecked can drive the localized intensity of the beam to levels capable of damaging expensive optical components. Under extreme conditions the beam is seen to evolve into small regions of extremely high intensity, a phenomenon known as filamentation. Invariably, the onset of these nonlinear processes constrains operators to limit the output of the system before the laser runs out of power.

For large fusion class lasers, the situation is exacerbated by economic forces which dictate that the laser be designed to operate as close to the damage threshold as possible. The cost of a large laser is strongly dependent on the size of its clear aperture, therefore one way of maximizing the performance to cost ratio is to make the aperture of the laser as small as possible. For a given output energy this naturally drives the fluence (energy per unit area) up. With this design strategy the goal is to reduce 
the aperture of the laser to the smallest area such that the fluence of the propagated pulse is below the damage threshold of the system. It is then necessary to make sure that the spatial profile of the beam remains as smooth as possible.

The National Ignition Facility (NIF) ${ }^{2}$ currently being designed at the Lawrence Livermore National Laboratory is the largest and most expensive laser system yet conceived. Achieving the design criteria of $1.8 \mathrm{MJ}$ in a $500 \mathrm{TW}, 20 \mathrm{~ns}$ pulse hinges on the laser's ability to propagate smooth, spatially uniform beams. Given the size, cost and complexity of the current design (192 independent beamlines of 1600 square centimeter aperture; total cost $\$ 1.1$ billion ) previous approaches to the control of nonlinear processes will have to be refined. In particular, the regular replacement of severely damaged optical components is not likely to be an option.

In this paper we describe our efforts to simulate and quantify the effects of beam breakup. In Section II we present some background material on the phenomenon of small scale self-focusing. Section III describes our experimental method for simulating NIF operating conditions on LLNL's two current high power solid state lasers, $\mathrm{Nova}^{3}$ and Beamlet $^{4}$. In Section IV a method for quantifying beam quality is presented based on statistics of the propagated beam. We present the results of our measurements and conclude that NIF redline operating conditions should be sufficient for safe operation.

\section{BACKGROUND}

The behavior of physical systems is generally determined by both the structure and dynamics of the system. In the study of the propagation of irregularities on high power laser beams, we are necessarily concerned with two quantities; the noise sources added to the beam, which determine the structure of the noise field, and their nonlinear power growth, which determines the dynamics. In short, the damage threat to the system is governed both by noise accumulation and by noise growth.

Typically, beam irregularities originate as imperfections on the surfaces of optical components such as lenses or amplifier slabs. These imperfections may be due to finishing marks, surface contamination or the residual effects of previous damage. When the laser pulse encounters such an imperfection, scattered waves are generated that prop- agate at some angle relative to the main beam. These scattered waves interfere with the main beam and each other, and the resulting interference pattern produces a modulation, or ripple, on the spatial profile of the main beam. Actual noise sources generally result in a complicated superposition of many such ripple patterns.

At high intensities nonlinear effects become important and the power contained in the scattered ripple waves can undergo gain. ${ }^{5,6}$ This gain is a consequence of the nonlinear, intensity dependent term in the index of refraction of most optical media. The intensity dependent term in the index is small making the effect all but undetectable with conventional sources. At intensities reached by high peak power solid state lasers the ripples resulting from the growth can dominate the spatial profile of the beam.

The quantity that is conventionally used to describe this nonlinear growth is the breakup- or $B$-integral:

$$
B=\frac{2 \pi}{\lambda} \int \gamma I(z) d z
$$

where $\lambda$ is the wavelength of the beam, $I$ is the intensity and $\gamma$ is the coefficient of the nonlinear term in the index of refraction. The greater the $B$-integral the greater the susceptibility of the system to the effects of self-focusing. Laser designers have generally had to learn what values of $B$ they can endure by experience. The matter is complicated by the fact that the spatial frequency components in the noise field grow at varying rates to yield an angularly dependent gain spectrum for the system. The upper limit for this gain is $e^{2 B}$. Since high power lasers accumulate $B$-integrals as high as 5 radians during propagation, the nonlinear gain of the fastest growing spatial frequencies can be of the order of tens of thousands.

The control of nonlinear ripple growth is accomplished through spatial filtering. The function of the filter is to re-initialize the portion of the noise field outside the acceptance angle of the filter pinhole to zero, thus limiting the nonlinear growth of that part of the spectrum to an amount given by the $B$-integral between pinholes. The proper choice of pinhole diameter depends on an accurate assessment of the nonlinear power growth spectrum. Fortunately, the gain spectrum can be derived using perturbation methods ${ }^{7}$ and can thus be calculated even for very complicated laser systems. Present fusion class laser systems typically operate with pin- 
hole acceptance angles in the neighborhood of 200 to $300 \mu \mathrm{rad}$. Attempting to reduce this angle produces a host of complications such as plasma induced pinhole closure, Gibbs modulation etc. Nonetheless, calculations indicate that non linear ripple growth and hence the damage threat to the NIF would be substantially reduced if pinholes with cut-off angles near $100 \mu \mathrm{rad}$ could be used.

\section{DESCRIPTION OF EXPERIMENTAL MEASUREMENTS}

We conducted a series of experiments on the Nova and Beamlet Lasers to examine the stressful conditions occurring at the end of long, temporally flat output pulses. This type of operation poses a large damage threat to the laser since high $B$-integrals can occur at the end of such pulses. The situation is illustrated schematically in Figure 1. During high energy operation, the tail-end of the pulse experiences reduced gain because of energy extraction. ${ }^{8,9}$ To produce a temporally flat output pulse, this effect must be compensated for by shaping the injected pulse so that its irradiance increases monotonically with time. The $B$-integral, being proportional to the irradiance, increases during the pulse, therefore the highest damage threat from nonlinear growth of modulation occurs at the end of the pulse where the amplifier gain is lowest and the $B$-integral is highest.
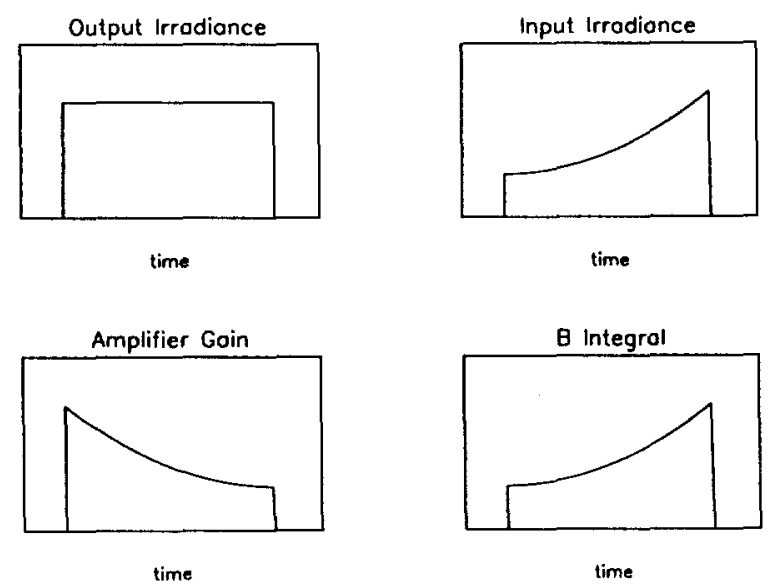

Figure 1. The conditions at the end of long, high energy, temporally flat output pulses can lead to a high damage threat for the laser.

To simulate the high- $B$ conditions at the end of the long saturating pulse, we propagated pulses of relatively short duration through the laser with reduced amplifier gains. The use of short pulses max- imizes our ability to accurately record beam modulation; long pulses are less suitable because the integrated modulation recorded by the near-field diagnostic time averages the incident beam's irradiance. The pulses used were Gaussian with durations of $200 \mathrm{ps}$ on Beamlet and $100 \mathrm{ps}$ on Nova.

The gains of the amplifiers were reduced to the saturated gain seen by the last photon in long energetic pulses. On Nova the gain of each amplifier stage can be adjusted independently. In our experiments, the gains corresponded to the conditions at the end of a 1 ns flat output pulse with an energy of $8 \mathrm{~kJ}$ at $8 \mathrm{TW}$. The multipass architecture of Beamlet (four pass cavity amplifier followed by a single pass booster amplifier each with an associated spatial filter) makes adjusting the gain more difficult because reducing the gain of the cavity affects all four passes. Therefore we conducted Beamlet tests with the final, booster, amplifier turned off. This approximates the conditions in which a pulse has extracted all the energy from the booster amplifiers.

In each case, we used a variety of spatial filter pinhole configurations to assess the effect of spatial filtering on beam quality. On Nova, we used two different pinholes in both the final and penultimate spatial filters. In the penultimate filter, pinhole diameters $1.5 \mathrm{~mm}$ and $3 \mathrm{~mm}$ were used to pass angles of $100 \mu \mathrm{rad}$ and $200 \mu \mathrm{rad}$ respectively. In the final filter, pinhole diameters were $1.8 \mathrm{~mm}(100 \mu \mathrm{rad}$ acceptance angle) and $4 \mathrm{~mm}$ ( $220 \mu \mathrm{rad})$. On Beamlet, we used pinhole acceptance angles of $100,130,150$, and $200 \mu \mathrm{rad}$ in the cavity filter and $100,130,150$ and $200 \mu \mathrm{rad}$ and fully removed in the booster spatial filter. Removing the booster spatial filter pinhole allows us to assess the modulation at the input lens of that filter.

\section{DATA}

Near-field images of beam irradiance obtained during the tests were analyzed by extracting a small subregion from the overall beam image and conducting a statistical analysis on the patch. This allowed us to choose the size and location of the subimage so as to avoid edge effects and to obtain a sample of the beam possessing a relatively constant background irradiance. Consequently, the statistical properties calculated from the patch are a property only of the modulation on the beam, not the non-uniformity of the background. For each patch we constructed a histogram giving the number of pixels occurring at each fluence, normalized so that the area under 
each curve is equal to one. Examples are shown in Figures 2 and 3 which contain the near-field images and histograms from a low power $1.57 \mathrm{GW} / \mathrm{cm}^{2}$ shot and a high power, $4.74 \mathrm{GW} / \mathrm{cm}^{2}$ shot. At low power, the spatial profile of the beam is generally, quite uniform and the histogram tends to be narrow and sharply peaked about the average fluence. As power increases, beam quality degrades; i.e. the irradiance modulation of the beam grows and the histogram tends to broaden and decrease in amplitude.

We found that the most robust figure of merit for assessing the beam quality was contrast ratio of the histogram, which measures the width, or the variation of the histogram about its mean. The contrast ratio is defined to be:

$$
c=\frac{1}{F_{\text {avg }}} \sqrt{\frac{\sum\left(F_{i}-F_{\text {avg }}\right)^{2}}{N}}
$$

where $F_{\text {avg }}$ is the average fluence of the patch analyzed, $N$ is the number of pixels in the patch and $F_{i}$ is the fluence of each pixel. This quantity was calculated for each patch analyzed. In general, beams with contrast ratios of 0.1 or less were found to have modest amounts of beam modulation.

\section{B5122709}
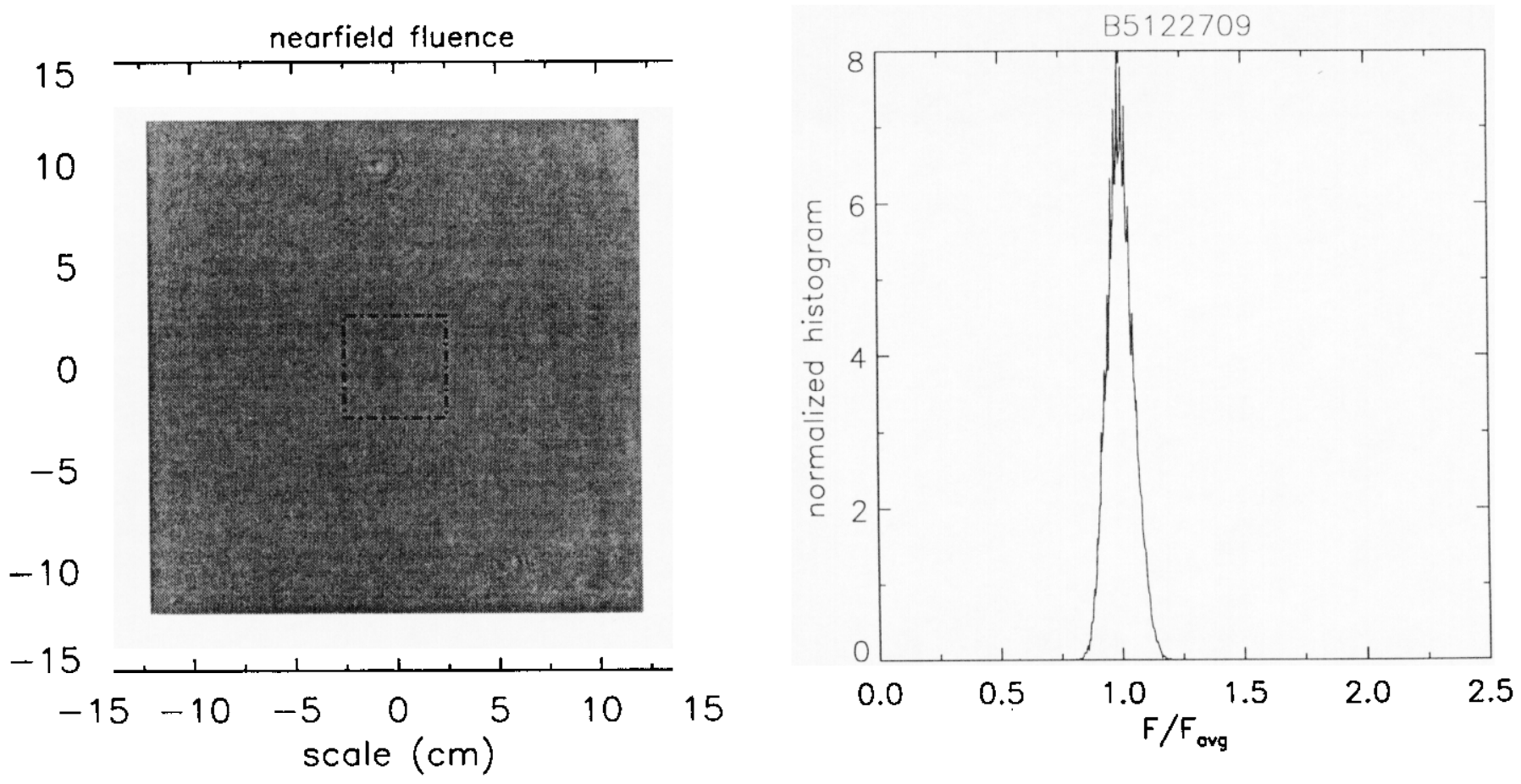

Figure 2. Near-field image and fluence histogram for a low power, $1.57 \mathrm{GW} / \mathrm{cm}^{2}$ shot on Beamlet. Patch used for statistical analysis is indicated. 

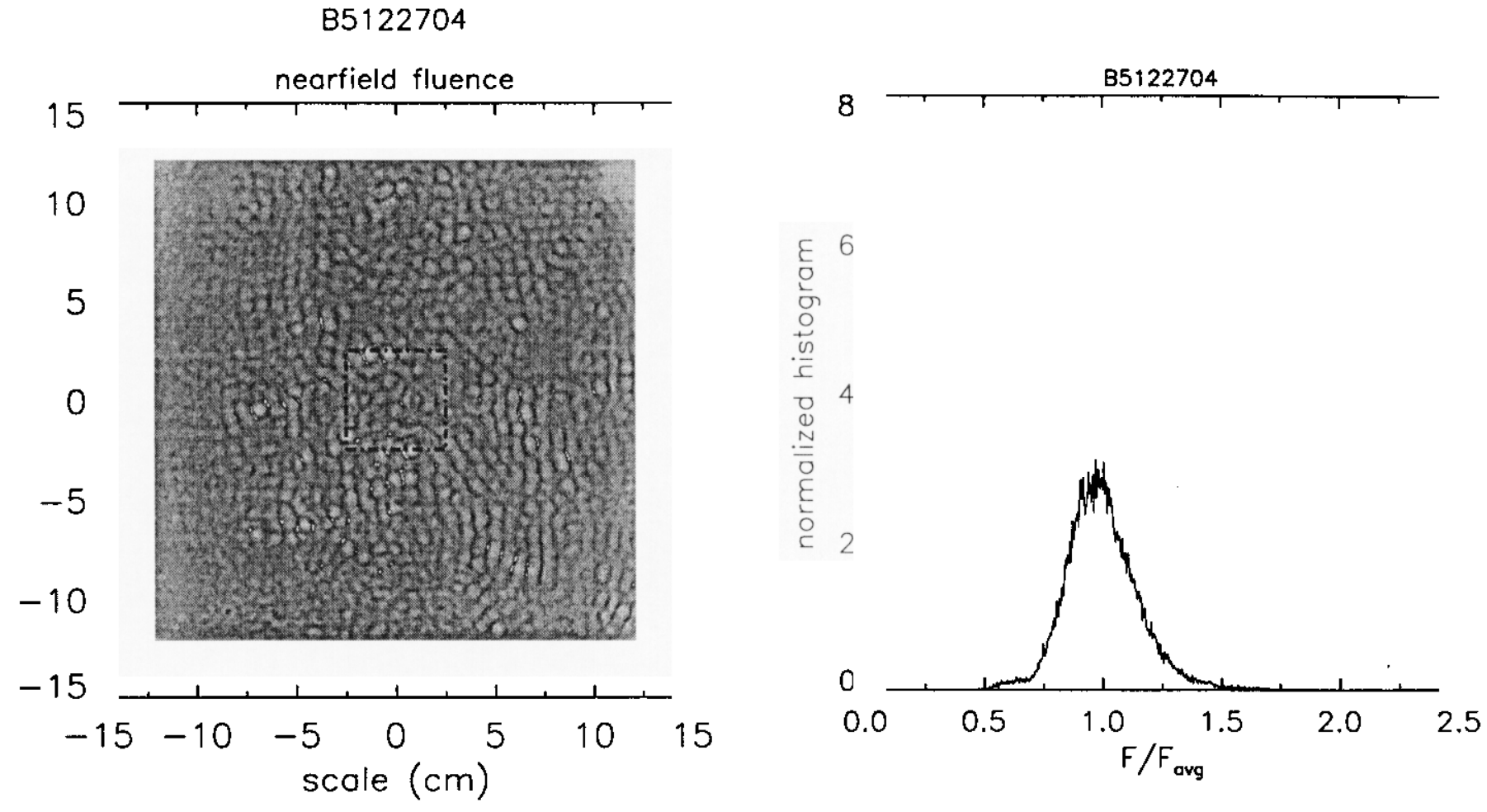

Figure 3. Near-field image and fluence histogram for a high power, $4.74 \mathrm{GW} / \mathrm{cm}^{2}$ shot on Beamlet. Patch used for statistical analysis is indicated.

\section{COMPARISON OF EXPERIMENT AND CALCULATION}

We calculated beam statistics for both the Beamlet and Nova shot data and compared these to numerical simulations using PROP92 ${ }^{10}$, a laser propagation code used at Livermore. Figure 4 compares the results for the Beamlet data obtained with $130 \mu \mathrm{rad}$ and $200 \mu \mathrm{rad}$ pinholes in the cavity filter. In each case the pinhole in the booster filter was open to allow us to assess the beam modulation at the input lens to the filter. The contrast ratio of the beam is plotted against the $B$-integral in the booster section of the laser.

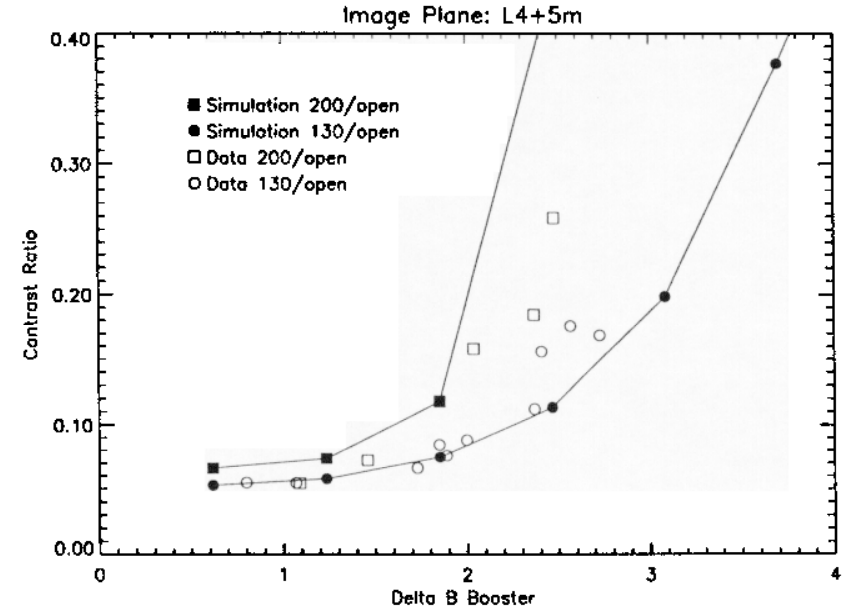

Figure 4. Contrast ratio vs $B$-integral for two pinhole configurations on Beamlet; $130 \mu \mathrm{rad} / \mathrm{open}$ and $200 \mu \mathrm{rad} /$ open. 
Open symbols in the plot represent shot data and solid points are the results of the numerical simulations. In each case circles represent the small pinhole configuration while the squares denote the larger $200 \mu \mathrm{rad}$ case. Notice that the onset of a rapid deterioration of beam quality occurs at a lower $B$ with the $200 \mu \mathrm{rad}$ cavity pinhole. At a $B$-integral of approximately 2.0 , the $130 \mu \mathrm{rad}$ data is clearly in the slowly varying portion of the curve. At the same value of $B$ however, the $200 \mu \mathrm{rad}$ data has begun a near vertical ascent towards beam breakup. It appears that for $B<2$, pinhole sizes up to $200 \mu \mathrm{rad}$ are acceptable, however, with the larger pinholes the margin for error is substantially reduced.

In Figure 5 we compare the Beamlet results for $130 / 100 \mu \mathrm{rad}$ and $200 / 200 \mu \mathrm{rad}$ cavity/booster pinholes. We attribute the lack of agreement between test data and simulation predictions in this figure to incomplete characterization of the noise fields in the 130 to $200 \mu \mathrm{rad}$ region. Note: the contrast ratio for the $130 / 100 \mu \mathrm{rad}$ pinholes remains small for values of $B$ up to 3 . This indicates that a smaller pinhole in the booster spatial filter should be effective in protecting the downstream optics.

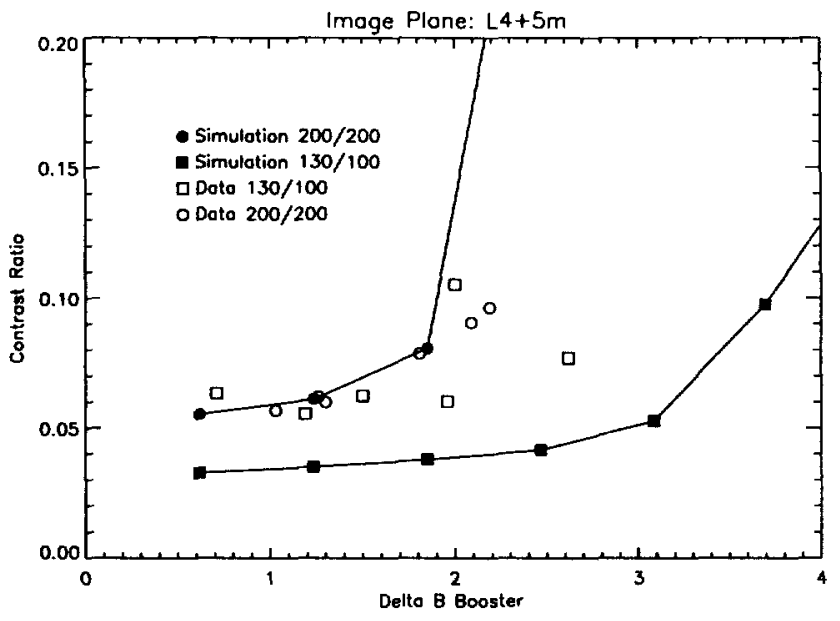

Figure 5. Contrast ratio vs $B$-integral for two pinhole configurations on Beamlet; $130 / 100 \mu \mathrm{rad}$ and $200 / 200 \mu \mathrm{rad}$.

Figure 6 shows the contrast ratio of the Nova beam as a function of the $B$-integral in the $\mathrm{f}$ nal amplifier section. Two pinhole configurations were used in the penultimate and final spatial filters respectively: $100 / 100 \mu \mathrm{rad}$ and $200 / 200 \mu \mathrm{rad}$. As with the Beamlet data, for $B<2$ operation with pinholes of $200 \mu \mathrm{rad}$ appears to be safe. However, the data with the smaller pinholes does not match the simulations very well and indeed is some- what counterintuitive in that the tighter filtering in the final and penultimate filters appears to reduce the threshold at which beam quality degrades. The Nova tests indicate that the near-field beam irradiance fluctuations can be increased with tighter filtering. We postulate that there are four possible noise sources in the Nova laser which could contribute to this effect: relatively large, low frequency source terms originating in the Nova preamplifier; large amplitude, low frequency phase errors that are either pump induced or thermally generated; irradiance nonuniformities produced by misalignment of the beam at the forming aperture or the pinholes, and whole beam self-focusing. Any of these sources could lead to near-field irradiance fluctuations whose frequency is centered at the bandpass of the penultimate filter and will experience nonlinear growth in the optics downstream from the filter. Further tests to measure the noise sources entering the final stages on Nova may provide some insight into this phenomena.

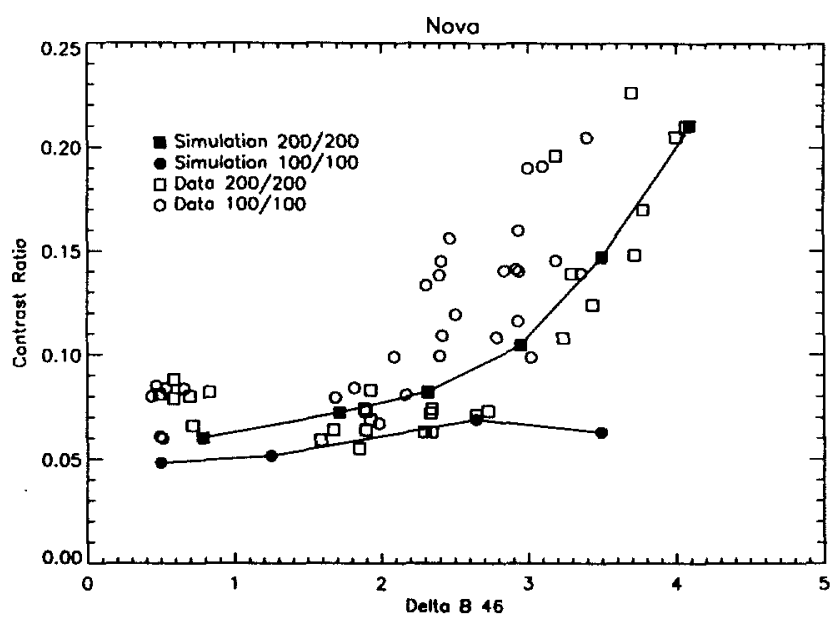

Figure 6. Contrast ratio vs $B$-integral for two pinhole configurations on Nova; $100 / 100 \mu \mathrm{rad}$ and $200 / 200 \mu \mathrm{rad}$.

\section{CONCLUSIONS}

In both the Beamlet and the Nova tests, for $B$ values of less than 1.8 radians (the NIF redline value) the contrast ratio has a value of less than 0.08 and, within error, is independent of the pinhole size. On Beamlet, as $B$ increases above 1.8 , the system with the smaller $130 / 100 \mu \mathrm{rad}$ pinholes shows a substantially slower rise in contrast ratio than those with the larger $200 / 200 \mu \mathrm{rad}$ pinholes. This result is in agreement with the modeling calculations and, in contradiction to the result obtained on Nova, indi- 
cates there are no large unidentified noise sources on Beamlet. The calculations follow the trends in the data but do not match them in detail. These discrepancies are not surprising considering our very incomplete knowledge of the noise sources. From the data we conclude that with Beamlet-quality optics and proper spatial filtering we should be able to propagate smooth, high quality beams at the NIF redline operating condition of $B \leq 1.8$ radians.

\section{ACKNOWLEDGMENTS}

Work performed under the auspices of the U.S. Department of Energy by the Lawrence Livermore National Laboratory under contract number W-7405ENG-48. The authors acknowledge J. B. Trenholme and K. R. Manes for many helpful discussions.

\section{REFERENCES}

1. V. I. Bespalov, V. I. Talanov. "Filamentary structure of light beams in nonlinear liquids," Zh. Eksp. Teor. Fiz. Pis'ma Red 3, 471-476 (1966) [JETP Lett. 3, 307 (1966)].

2. "National Ignition Facility Conceptual Design Report," 2 and 3, Rep. UCRL-PROP-117903 (Lawrence Livermore National Laboratory, Livermore, Calif., 1994).

3. J. T. Hunt, D. R. Speck, "Present and Future Performance of the Nova Laser System," Opt. Eng. 28, 461-468 (1989).

4. B. M. Van Wonterghem, J. R. Murray, J. H. Campbell, D. R. Speck, C. E. Barker, I. C. Smith, D. F. Browning, W. C. Behrendt, "System Description and Initial Performance Results for Beamlet," ICF Quarterly Report, 5(1), 1-17 (Lawrence Livermore National Laboratory, 1994).

5. E. S. Bliss, D. R. Speck, J. F. Holzrichter, J. H. Erkkila, A. J. Glass, "Propagation of a high-intensity laser pulse with a small scale intensity modulation," Appl. Phys. Lett. 25, 448-450 (1974).

6. J. A. Fleck, J. R. Morris, E. S. Bliss, "Small scale self-focusing effects in a high-power glass laser amplifier," IEEE J. Quantum Electron. QE-14, 353-363 (1978).
7. J. B. Trenholme, "Theory of irregularity growth on laser beams," 1975 Laser Annual Report, Rep. UCRL-50021-75, 237-242 (Lawrence Livermore National Laboratory, 1975).

8. L. M. Frantz, J. S. Nodvik, "Theory of pulse propagation in a laser amplifier," $J$. Appl. Phys. 34(8), 2346-2349 (1963).

9. J. B. Trenholme, K. R. Manes, "A simple approach to laser amplifiers," Rep. UCRL51413 (Lawrence Livermore National Laboratory, 1972).

10. PROP92 is a two-dimensional FFT laser propagation code originally developed at LLNL by Robert G. Nelson and enhanced and modified by Richard A. Sacks, Scott W. Haney and Mark A. Henesian. In addition to modeling the performance of the Beamlet and Nova lasers, PROP92 simulations were used to determine the optimized NIF baseline design. 


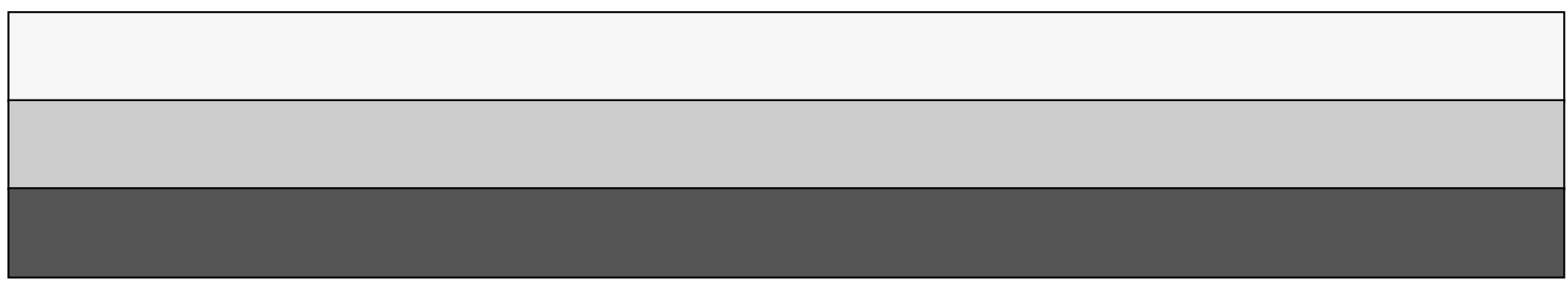

